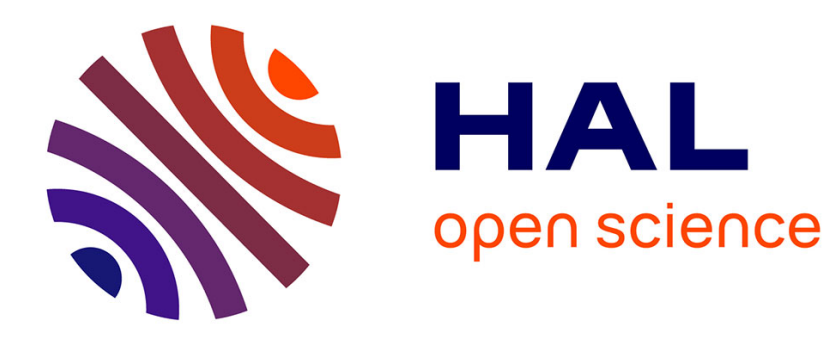

\title{
From frustrationless models to spin glasses
} J.S. Denbigh

\section{To cite this version:}

J.S. Denbigh. From frustrationless models to spin glasses. Journal de Physique Lettres, 1980, 41 (1), pp.13-18. 10.1051/jphyslet:0198000410101300 . jpa-00231707

\section{HAL Id: jpa-00231707 https://hal.science/jpa-00231707}

Submitted on 1 Jan 1980

HAL is a multi-disciplinary open access archive for the deposit and dissemination of scientific research documents, whether they are published or not. The documents may come from teaching and research institutions in France or abroad, or from public or private research centers.
L'archive ouverte pluridisciplinaire HAL, est destinée au dépôt et à la diffusion de documents scientifiques de niveau recherche, publiés ou non, émanant des établissements d'enseignement et de recherche français ou étrangers, des laboratoires publics ou privés. 


\title{
From frustrationless models to spin glasses
}

\author{
J. S. Denbigh \\ Physics Department, Imperial College, London S.W.7, U.K. \\ (Reçu le 20 août 1979, révisé le 30 octobre 1979, accepté le 15 novembre 1979)
}

\begin{abstract}
Résumé. - On compare le comportement du modèle de Mattis en champ nul au-dessous de la température critique avec celui d'un superparamagnétique de moment d'ordre $N^{1 / 2}$ où $N$ est le nombre de sites. On discute l'effet des moments superparamagnétiques dans les modèles avec frustration et l'on montre comment les résultats récents de Monte Carlo sont compatibles avec un pic aigu de la susceptibilité mesurée.
\end{abstract}

Abstract. - The behaviour of a Mattis model in zero field below the critical temperature is compared with that of a superparamagnet with moment of order $N^{1 / 2}$. The effect of superparamagnetic moments in models with frustration is discussed and it is shown how recent Monte Carlo results may be consistent with a cusp in the measured susceptibility.

1. Frustrationless models. - Consider a hamiltonian of form

$$
H=-\sum_{(i j)} \varepsilon_{i j} J_{i j} \mathbf{s}_{i} \cdot \mathbf{A} \cdot \mathbf{s}_{j}-B \sum_{i=1}^{N} s_{i z} .
$$

(ij) is any pair of sites, there being $N$ sites altogether. $\mathbf{s}_{i}, \mathbf{s}_{j}$ represent the spins of the $i$ th and $j$ th sites and their components, $s_{i x}, s_{j x}, s_{i y}$ etc. are assumed to commute. $A$ is a symmetric tensor which determines whether the interactions are Ising, Heisenberg etc. and which is normalised so that its largest eigenvalue is 1 . $J_{i j}$ represents the strength of the interaction between the $i$ th and $j$ th sites and $\varepsilon_{i j}$ its sign. $J_{i j}$ is assumed to depend only on $\left|\mathbf{r}_{i}-\mathbf{r}_{j}\right|$. $B$ represents an external magnetic field parallel to $O Z$.

A model is called frustrationless when in the absence of a field there is a ground state $\left|{ }_{0} \mathbf{S}\right\rangle=\left|{ }_{0} \mathbf{s}_{1}\right\rangle\left|{ }_{0} \mathbf{s}_{2}\right\rangle\left|{ }_{0} \mathbf{s}_{N}\right\rangle$ such that each bond has its lowest possible energy. The lowest possible value of the energy of the bond between the $i$ th and $j$ th sites is $-J_{i j}$. This occurs when $\mathbf{s}_{i}=\varepsilon_{i} \mathbf{e}, \mathbf{s}_{j}=\varepsilon_{j} \mathbf{e}$ where $\mathbf{A e}=\mathbf{e}, \varepsilon_{i}= \pm 1$ and $\varepsilon_{j}= \pm 1$. It follows that

$$
\varepsilon_{i j}=\varepsilon_{i} \varepsilon_{j} .
$$

The $\varepsilon_{i j}$ can therefore be expressed in terms of just $N$ variables. In this letter we shall only be interested in cases where a bond is as likely to be ferromagnetic as antiferromagnetic. This condition can be met by making each $\varepsilon_{i}$ randomly \pm 1 . Let the set $\left\{\varepsilon_{i}\right\}$ be denoted by $\boldsymbol{\varepsilon}$. Putting $\tau_{i}=\varepsilon_{i} \mathbf{s}_{i}$

$$
H=-\sum_{i j} J_{i j} \tau_{i} \cdot \mathbf{A} \cdot \tau_{j}-B \sum_{i=1}^{N} \varepsilon_{i} \tau_{i z} .
$$

In zero field this hamiltonian is the same as that of a ferromagnet so the zero field specific heat behaves in exactly the same way as that of the corresponding ferromagnetic model. This means that when the dimensionality is greater than 1 for the Ising model and 2 for the Heisenberg model there is a critical temperature, $T_{\mathrm{f}}$ below which

$$
\lim _{\left|r_{i}-r_{j}\right| \rightarrow \infty}\left\langle\tau_{i z} \tau_{j z}\right\rangle
$$

is non-zero, $=\mu^{2}$ say. The treatment of this model by [1] and [2] assumes that $\left\langle\tau_{i}\right\rangle=\mu$ for $T<T_{\mathrm{f}}$ at $B=0$. It is more correct to assume below $T_{\mathrm{f}}$ that $\lim _{B \rightarrow 0} \lim _{N \rightarrow \infty}\left\langle\tau_{i}\right\rangle=\mu$ but that for any finite $N\left\langle\tau_{i}\right\rangle$ is zero at any temperature because for any state there is a state with the same energy with all spins reversed. This means that below we consider the complete ensemble of states and the possibility that all spins may reverse directions. [1, 2, 3], assume symmetry breaking.

$M$, the magnetisation in the $O Z$ direction is $\sum_{i=1}^{N} \varepsilon_{i} \tau_{i z}$. For any operator, $A$, let $\langle A\rangle$ represent 
the thermodynamic average, $\operatorname{tr}\left(A \mathrm{e}^{-\beta H}\right) / \operatorname{tr}\left(\mathrm{e}^{-\beta H}\right)$, $\beta$ being $(k T)^{-1}$. For any $n$

$$
\frac{\partial}{\partial B}\left\langle M^{n}\right\rangle=\beta\left(\left\langle M^{n+1}\right\rangle-\left\langle M^{n}\right\rangle\langle M\rangle\right) .
$$

It should be noticed that $M$ is $M(\varepsilon)$, dependent on the particular set $\varepsilon$ and likewise $\chi$, the susceptibility/site is $\chi(\varepsilon)=N^{-1} \frac{\partial}{\partial B}\langle M\rangle$. Using eq. (2)

$$
\begin{aligned}
\chi & =N^{-1} \beta\left(\left\langle M^{2}\right\rangle-\langle M\rangle^{2}\right) \\
\frac{\partial^{2} \chi}{\partial B^{2}} & =N^{-1} \beta^{3}\left(\left\langle M^{4}\right\rangle-3\left\langle M^{2}\right\rangle^{2}-4\left\langle M^{3}\right\rangle\langle M\rangle+12\left\langle M^{2}\right\rangle\langle M\rangle^{2}-6\langle M\rangle^{4}\right) .
\end{aligned}
$$

At zero field $\langle M\rangle=0$ as $N$ is finite so from eq. (3)

$$
\chi(0)=N^{-1} \beta \sum_{i j} \varepsilon_{i} \varepsilon_{j}\left\langle\tau_{i z} \tau_{j z}\right\rangle .
$$

Let $\langle\bar{M}\rangle$ and $\bar{\chi}$ be averages taken over the $2^{N}$ possible $\varepsilon$ so that

$$
\langle\bar{M}\rangle=2^{-N} \sum_{\varepsilon}\langle M(\varepsilon)\rangle, \quad \bar{\chi}=2^{-N} \sum_{\varepsilon} \chi(\varepsilon) .
$$

From eq. (4)

$$
\begin{gathered}
\bar{\chi}(0)=N^{-1} \beta \sum_{i=1}^{N}\left\langle\tau_{i z}^{2}\right\rangle,=\left\{\begin{array}{l}
\beta \text { for Ising spin } 1 / 2 \\
1 / 3 \beta \text { for classical Heisenberg }
\end{array}\right. \\
\frac{\partial^{2}}{\partial B^{2}} \bar{\chi}(0)=N^{-1} \beta^{3} \sum_{i=1}^{N}\left(\left\langle\tau_{i z}^{4}\right\rangle-3\left\langle\tau_{i z}^{2}\right\rangle^{2}\right)+3 N^{-1} \beta^{3} \sum_{i \neq j}\left\{\left(\left\langle\tau_{i z}^{2} \tau_{j z}^{2}\right\rangle-\left\langle\tau_{i z}^{2}\right\rangle\left\langle\tau_{j z}^{2}\right\rangle\right)-2\left\langle\tau_{i z} \tau_{j z}\right\rangle\right\}^{2} .
\end{gathered}
$$

Above $T_{\mathrm{f}}$ both

$$
\left\langle\tau_{i z} \tau_{j z}\right\rangle \text { and }\left(\left\langle\tau_{i z}^{2} \tau_{j z}^{2}\right\rangle-\left\langle\tau_{i z}^{2}\right\rangle\left\langle\tau_{j z}^{2}\right\rangle\right),
$$

being correlation functions are expected to decay exponentially with $\left|\mathbf{r}_{i}-\mathbf{r}_{j}\right|$ so as

$$
N \rightarrow \infty \frac{\partial^{2}}{\partial B^{2}} \bar{\chi}(0)
$$

tends to a finite limit. For $T<T_{\mathrm{f}}$ however $\lim _{\left|\mathbf{r}_{i}-\mathbf{r}_{j}\right| \rightarrow \infty}\left\langle\tau_{i z} \tau_{j z}\right\rangle$ is non zero and $\frac{\partial^{2}}{\partial B^{2}} \bar{\chi}(0)$ diverges as $0(N)$. These results are generalisations of those obtained by Mattis [4] for the Ising spin 1/2 case.

If $m_{0}$ is the spontaneous magnetisation of the corresponding ferromagnet $\mu=m_{0}$ in the Ising spin $1 / 2$ case and $3^{-1 / 2} m_{0}$ in the classical Heisenberg case. Let $N^{-1 / 2} \sum_{i=1} \varepsilon_{i}=u(\varepsilon)$. As $N \rightarrow \infty$ the probability, $P(U) \mathrm{d} U$ that $U<u(\varepsilon)<U+\mathrm{d} U$ for a randomly picked $\varepsilon$ is gaussian with variance equal to one. From eq. (4)

$$
\begin{array}{r}
\chi(0)=\beta \mu^{2} u^{2}(\varepsilon)+\beta N^{-1} \sum_{i=1}\left(\left\langle\tau_{i z}^{2}\right\rangle-\mu^{2}\right)+ \\
+\beta N^{-1} \sum_{i \neq j} \varepsilon_{i} \varepsilon_{j}\left(\left\langle\tau_{i z} \tau_{j z}\right\rangle-\mu^{2}\right) .
\end{array}
$$

For a randomly picked $\varepsilon$ the last term tends to zero as $N \rightarrow \infty$ at any temperature. The second term tends to a non zero limit as $N \rightarrow \infty$. For $T>T_{\mathrm{f}}$ the first term is zero as $\mu$ is zero. For $T<T_{\mathrm{f}}$ the first term being dependent on $u(\varepsilon)$ varies from one $\varepsilon$ to another, the spread of values being of the order $\beta \mu^{2}$. When the field is exactly zero the susceptibility has no thermodynamic limit although it remains finite as $N \rightarrow \infty$.

2. The physical interpretation. - There is a simple intuitive explanation for these results. Below the critical temperature the relative directions of the spins are nearly fixed so that for some each $\mathbf{s}_{i}$ is usually close to $\varepsilon_{i} \mathbf{e}$. The total magnetic moment is approximately $m_{0} \sum_{i=1}^{N} \varepsilon_{i} \mathbf{e}=m_{0} N^{1 / 2} u(\varepsilon) \mathbf{e}, m_{0}$ being the sponteneous magnetisation/site. Let $\chi_{\mathrm{s}}(B)$ be $N^{-1} x$ the susceptibility of an isolated superparamagnet with this moment in field $B$. In the Ising spin $1 / 2$ case or where there is a potential barrier this magnet will be assumed to have two states as e takes the values $\pm O Z$. In the classical Heisenberg case this magnet will be free to rotate in three dimensions as e can now be any direction. For certain interactions a rotation in just two dimensions is permitted. Let

$$
\chi(B)=\chi_{\mathrm{s}}(B)+\chi_{\mathrm{m}}(B)
$$

$\chi_{\mathrm{s}}(B)$ is as defined above and is the superparamagnetic component of $\chi(B) \cdot \chi_{\mathrm{m}}(B)$ is due to the effect the field has on increasing correlations between spins. $\chi_{\mathrm{s}}(0)=\beta \mu^{2} u^{2}(\varepsilon)$. For large $N$

$$
\chi_{\mathrm{m}}(0)=\beta N^{-1} \sum_{i=1}^{N}\left(\left\langle\tau_{i z}^{2}\right\rangle-\mu^{2}\right)
$$

in order that eq. (7) agrees with eq. (6). 
Let $\left\langle M_{\mathrm{s}}\right\rangle$ be the thermodynamic average magnetisation of the superparamagnet. If $B_{\mathrm{s}}$ is the appropriate Brillouin function the usual theory gives

$$
\begin{aligned}
\left\langle M_{\mathrm{s}}\right\rangle & =m_{0} u N^{1 / 2} B_{\mathrm{s}}\left(m_{0} u N^{1 / 2} \beta B\right), \\
\frac{\partial^{2 j}}{\hat{c} B^{2 j}} \chi_{\mathrm{s}}(0) & =\left(m_{0} u\right)^{2 j+2_{\beta} 2 j+1} N^{j} \frac{\partial^{2 j+1}}{\partial x^{2 j+1}} B_{\mathrm{s}}(x)_{x=0} \quad \text { for } \quad j \geqslant 0 .
\end{aligned}
$$

Below $T_{\mathrm{f}} \chi_{\mathrm{s}}(0)$ and $\bar{\chi}(0)$ and their higher derivatives depend on $N$ and $\beta$ in a similar way. $\frac{\partial^{2}}{\partial B^{2}} \bar{\chi}(0)$ has been shown to diverge like $N$ as $N \rightarrow \infty$ and unless terms cancel in an unexpected way $\frac{\partial^{2 j}}{\partial B^{2 j}} \bar{\chi}(0)$ diverges as $0\left(N^{j}\right)$ for $j>1$.

Because the moment of the superparamagnet is $0\left(N^{1 / 2}\right)$ whatever $b$ is $\int_{-b}^{b} \chi_{s}(B) \mathrm{d} B$ does not exceed $0\left(N^{-1 / 2}\right)$. Eq. (9) shows that if $B>0\left(N^{-1 / 2}\right)$ the magnetisation is saturated and $\chi_{\mathrm{s}}(B)$ negligible. Suppose a frustrationless model could be realised physically and its susceptibility estimated by measuring

$$
1 / 2 b^{-1}\{\langle m(b)\rangle-\langle m(-b)\rangle\} \text {. }
$$

The contribution due to $\chi_{\mathrm{s}}$ would be negligible unless $b$ were $0\left(N^{-1 / 2}\right)$ or less. The measurable part of $\chi(B)$ is therefore $\chi_{\mathrm{m}}(B)$.

If $B$ is not necessarily assumed to be zero from eq. (3)

$$
\chi(B)=N^{-1} \beta \sum_{i}\left(\left\langle\tau_{i z}^{2}\right\rangle-\left\langle\tau_{i z}\right\rangle^{2}\right)+N^{-1} \beta \sum_{i \neq j} \varepsilon_{i} \varepsilon_{j}\left(\left\langle\tau_{i z} \tau_{j z}\right\rangle-\left\langle\tau_{i z}\right\rangle\left\langle\tau_{j z}\right\rangle\right) .
$$

$\left\langle\tau_{i z}\right\rangle \neq 0$ if $B \neq 0$ and is correlated to $\varepsilon_{i}$. If $B \neq 0 \lim _{N \rightarrow \infty}\left(\left\langle\tau_{i z} \tau_{j z}\right\rangle-\left\langle\tau_{i z}\right\rangle\left\langle\tau_{j z}\right\rangle\right)$ decays exponentially with $\left|\mathbf{r}_{i}-\mathbf{r}_{j}\right|$. This means that $\lim _{N \rightarrow \infty} \chi(B)$ exists and is $\lim _{N \rightarrow \infty} \bar{\chi}(B) . \lim _{N \rightarrow \infty} \chi(B)=\lim _{N \rightarrow \infty} \chi_{\mathrm{m}}(B)$ if $B \neq 0$ since $\lim _{N \rightarrow \infty} \chi_{\mathrm{s}}(B)=0 . \lim _{B \rightarrow 0+} \lim _{N \rightarrow \infty}\left\langle\tau_{i z}\right\rangle \stackrel{N \rightarrow \infty}{=} \mu . \lim _{B \rightarrow 0+} \lim _{N \rightarrow \infty} \sum_{i \neq j} \varepsilon_{i} \varepsilon_{j}\left(\left\langle\tau_{i z} \tau_{j z}\right\rangle-\left\langle\tau_{i z}\right\rangle\left\langle\tau_{j z}\right\rangle\right)$ is zero for as $B$ tends to zero $\tau_{i z}$ cases to be correlated to $\varepsilon_{i}$.

$$
\begin{aligned}
\lim _{B \rightarrow 0} \lim _{N \rightarrow \infty} \chi_{\mathrm{m}}(B) & =\beta\left(\left\langle\tau_{i z}^{2}\right\rangle-\mu^{2}\right) & & \text { by eq. (11) } \\
& =\chi_{\mathrm{m}}(0) & & \text { by eq. (8) }
\end{aligned}
$$

$\chi_{\mathrm{m}}(B)$ is therefore expected to be a smooth function of $B$ expect when $T=T_{\mathrm{f}}$.

Eq. (8) shows that above $T_{\mathrm{f}}$ where $\mu$ is zero $\chi_{\mathrm{m}}(0)$ obeys Curie's law. It is essentially paramagnetic being independent of the $J_{i j}$ in both the Ising spin $1 / 2$ and classical Heisenberg cases since $\left\langle\tau_{i z}^{2}\right\rangle$ is independent of temperature. Just below $T_{\mathrm{f}} \chi_{\mathrm{m}}(0)$ has a critical exponent equal to the square of the exponent for the spontaneous magnetisation. It has an asymmetric cusp at $T_{\mathrm{f}}$. At very low temperatures $\chi_{\mathrm{m}}(0)$ behaves like $\mathrm{e}^{-\beta J}$ for some $J . \chi_{\mathrm{m}}$ is obtained directly from eq. (6) by assuming $\sum_{i=1}^{N} \varepsilon_{i}$ is zero. This assumption was made by Luttinger [5] when he examined a mean field Ising spin $1 / 2$ frustrationless model. Using the hamiltonian

$$
H=-(2 N)^{-1} \sum_{(i j)}\left(J_{1}+J_{2} \varepsilon_{i} \varepsilon_{j}\right) s_{i} s_{j}-B \sum_{i=1}^{N} s_{i}
$$

he finds that if $J_{2}>J_{1}$ there is a critical temperature $T_{\mathrm{f}}$ where the specific heat has a finite jump. The susceptibility has a cusp at $T_{\mathrm{f}}$ and in this neighbourhood for some positive $a, b$ independent of $T$

$$
\chi= \begin{cases}a-b\left(T-T_{\mathrm{f}}\right), & T>T_{\mathrm{f}} \\ a-2 b\left(T_{\mathrm{f}}-T\right), & T<T_{\mathrm{f}} .\end{cases}
$$

Presumably because $\sum_{i=1}^{N} \varepsilon_{i}=0$ in his results the superparamagnetic part is automatically eliminated. The cusp he obtains is less asymmetric than that obtained using nearest neighbour interactions.

Using results for the three dimensional Heisenberg ferromagnet given in Rushbrooke et al. [6] for the frustrationless Heisenberg model the specific heat exponent, $\alpha$ should be about -0.1 and the low temperature susceptibility exponent, $\gamma^{\prime}$ about $2 / 3$. If there are only interactions between nearest neighbours 
and $S(T)$ is the entropy at temperature $T$, $\left\{S\left(T_{\mathrm{f}}\right)-S(0)\right\}\{S(\infty)-S(0)\}^{-1}$ should be about $2 / 3$ and greater when longer range interactions are included. Experimental values of $\left\{S\left(T_{\mathrm{f}}\right)-S(0)\right\}$ $\{S(\infty)-S(0)\}^{-1}$ are about $1 / 3$ for $\mathrm{CuMn}$ and $\mathrm{FeAu}$ spin glasses [7]. Experimentally the cusp in $\chi(0)$ is nearly symmetric so the frustrationless model does not correspond very closely with experiment.

3. More general models. - It is possible that more general models of spin glasses with frustration and with equal numbers of ferromagnetic and antiferromagnetic bonds behave superparamagnetically below $T_{\mathrm{f}}$. We assume that there is a freezing temperature, $T_{\mathrm{f}}$ below which the Edwards and Anderson [8] order parameter is non zero. Such a system below $T_{\mathrm{f}}$ has a superparamagnetic moment of order $N^{1 / 2}$ which vanishes above $T_{\mathrm{f}}$. Eq. (7) can be applied and we conjecture that $\chi_{\mathrm{m}}(B)$ is a smooth function of $B$.

In order to estimate the superparamagnetic moment consider a model whose bond distribution is specified by $\left\{J_{i j}\right\} \equiv \mathbf{J}$, say starting at some initial state $\left|\mathrm{S}_{0}\right\rangle$ and in contact with a heat bath at temperature, $T$. The state of the system, $|\mathrm{S}(t)\rangle$ varies with time, $t$ and we shall consider

$$
\mathbf{r}_{i}\left(\mathbf{J}, \mathrm{S}_{0}, T\right) \equiv t^{-1} \int_{t^{\prime}=0}^{t} \mathbf{s}_{i}\left(t^{\prime}\right) \mathrm{d} t^{\prime}
$$

where $t$ is assumed to be very large. In a finite size system after a sufficiently long time a state is reached where all spins are reversed so that $\mathbf{r}_{i}$ is always zero for sufficiently large $t$ [9].

However let us define $\left|S^{\prime}(t)\right\rangle$ to be obtained by applying the same rotation to each spin in $|S(t)\rangle$ such that now $\sum_{i=1}^{N} s_{i}^{\prime}(t)$ lies along the positive $O Z$ axis. $\left|\mathbf{S}^{\prime}(t)\right\rangle$ is now relabelled $|\mathrm{S}(t)\rangle$. If there is no freezing $\mathbf{r}_{i}$ is $0\left(N^{-1 / 2}\right)$ but with freezing $r_{i}$ is $0(1)$. $r_{i}$ is independent of $\left|S_{0}\right\rangle$ in a finite size system and infinite $t$ although when $N$ is large the equilibrium value may take a very long time to achieve. As $N \rightarrow \infty \mathbf{r}_{i}$ may become dependent on $\left|\mathbf{S}_{0}\right\rangle$ although this is not so in the case of a pure ferromagnet or a frustrationless model at very low temperatures. Freezing does not necessarily imply the system is not in equilibrium.

The superparamagnetic moment of the entire system, $\mathbf{M}_{\mathrm{s}}$ is defined to be $\sum_{i=1}^{N} \mathbf{r}_{i}\left(\mathbf{J}, \mathrm{S}_{0}, T\right)$. Let \langle\rangle indicate a thermal average overall $\left|S_{0}\right\rangle$ and a indicate an average overall possible $\mathbf{J}$. Let $\mathbf{J}^{\prime},\left|\mathbf{S}_{0}^{\prime}\right\rangle$ be the same as $\mathbf{J},\left|\mathbf{S}_{0}\right\rangle$ except that the $i$ th spin has been reversed and the signs of all the bonds attached to it have been reversed. $\mathbf{r}_{i}\left(\mathbf{J}^{\prime}, S_{0}^{\prime}, T\right)$ equals $-\mathbf{r}_{i}\left(\mathbf{J}, \mathrm{S}_{0}, T\right)$ so that $\left\langle\overline{\mathbf{r}_{i}\left(J, \mathrm{~S}_{0}, T\right) \cdot \mathbf{r}_{j}\left(J, \mathrm{~S}_{0}, T\right.}\right\rangle$ is zero if $i \neq j$. Hence

$$
\overline{\left\langle M_{\mathrm{s}}^{2}(T)\right\rangle}=\sum_{i=1}^{N}\left\langle\overline{\mathbf{r}_{i}^{2}\left(\mathbf{J}, \mathrm{S}_{0}, T\right)}\right\rangle \equiv N q(T),
$$

$q(T)$ being indentical with the Edwards and Anderson order parameter. $N^{1 / 2} q^{1 / 2}(T)$ is therefore the average moment of the superparamagnet. As in the case of the frustrationless model we assume it either has two states, up or down or else is free to rotate. $\overline{\chi_{\mathrm{s}}}(0)$ is $\beta q(T)$ for the Ising spin $1 / 2$ case and $1 / 3 \beta q(T)$ for the classical Heisenberg case.

Domb [10] proved the Curie law for an Ising spin 1/2 model using a high temperature series expansion. More generally,

$$
\begin{gathered}
\overline{\chi(0)}=N^{-1} \beta \sum_{i j}\left\langle\overline{s_{i z} s_{j z}}\right\rangle=\beta\left\langle\overline{s_{i z}^{2}}\right\rangle . \\
\overline{\chi_{\mathrm{m}}(0)}=\overline{\chi(0)}-\overline{\chi_{\mathrm{s}}(0)}= \begin{cases}\beta(1-q), & \text { Ising spin } 1 / 2 \\
1 / 3 \beta(1-q), & \text { classical }\end{cases} \\
\text { Heisenberg. }
\end{gathered}
$$

This expression for $\overline{\chi_{\mathrm{m}}(0)}$ is identical with the expression for $\overline{\chi(0)}$ obtained by Edwards and Anderson [8] and Chalupa [3]. The derivation of $\overline{\chi_{m}(0)}$ above is similar to Chalupa's derivation of $\chi(0)$. However in [3] and [1] it is assumed that $\left\langle s_{i}\right\rangle$ is non zero. This means they have considered $\lim _{B \rightarrow 0} \lim _{N \rightarrow \infty} \chi(B)$ which turns out to be the same as $\frac{B \rightarrow 0_{+}}{\chi_{\mathrm{m}}(0)}$.

The above theory is consistent with the Monte Carlo results of Bray and Moore [11] on a $50 \times 50$ square lattice with nearest neighbour Ising interactions. Let

$\chi_{t}=N^{-1} \beta\left\{t^{-1} \int_{0}^{t} M^{2}\left(t^{\prime}\right) \mathrm{d} t^{\prime}-t^{-2}\left(\int_{0}^{t} M\left(t^{\prime}\right) \mathrm{d} t^{\prime}\right)^{2}\right\}$

where $t$ can be identified either with time or the number of Monte Carlo steps/site. At a temperature we estimate to be slightly below $T_{\mathrm{f}}$ they find that $\chi_{t}$ plotted against $t$ is a curve with sharp zigzags which tends asymptotically to $\beta$. This can be explained by assuming $M$ reverses direction periodically so that

$$
t^{-1} \int_{0}^{t} M\left(t^{\prime}\right) \mathrm{d} t^{\prime} \rightarrow 0
$$

as $t \rightarrow \infty$ leaving just the first term in eq. (14) which gives Curie's law. Reversals of $M$ are of course less probable at a lower temperature or on a larger lattice or in a higher dimension. This is illustrated by the Monte Carlo results of Bray, Moore and Reed [12] on a $10 \times 10 \times 10$ Ising lattice. In a Mattis model, which is equivalent to a ferromagnet reversals of $M$ are much less probable since each spin knows clearly which way to point and the energy required to flip a large cluster of spins is much higher. This is again shown by their results. If the observation time is sufficiently long however, any finite lattice must give Curie's law. This does not imply the absence of freezing, however, for this is known to exist in the Mattis model.

The Monte Carlo results of Bray et al., Binder 
and Rapaport [11-15] indicate that $\chi_{t}(0)$ as $N \rightarrow \infty$ is not Curie - like as temperature tends to the absolute zero. This means that either the system developes a high viscosity at low temperatures preventing equilibrium from being reached during the life of the experiment or there is a phase transition or both. We have just suggested that the results of Bray and Moore are consistent with a phase transition. The experimental evidence $[16,17]$ seems to indicate a sharp peak in the susceptibility at some temperature which appears to show little dependence on $\omega$, the frequency of the magnetic field used in the measurement. Even if the peak turns out not to be perfectly sharp it is sharp enough to suggest there should be some idealised model close to physical reality which does have a cusp.

Numerical results $[18,19]$ suggest the existence of defect surfaces on either side of which one may reverse all the spins without significantly increasing the ground state energy. Reed et al. [19] have also suggested that round any point there are defect surfaces of as great a perimeter as one likes when $N$ is infinite. This is illustrated in figure 1. At a suffi-

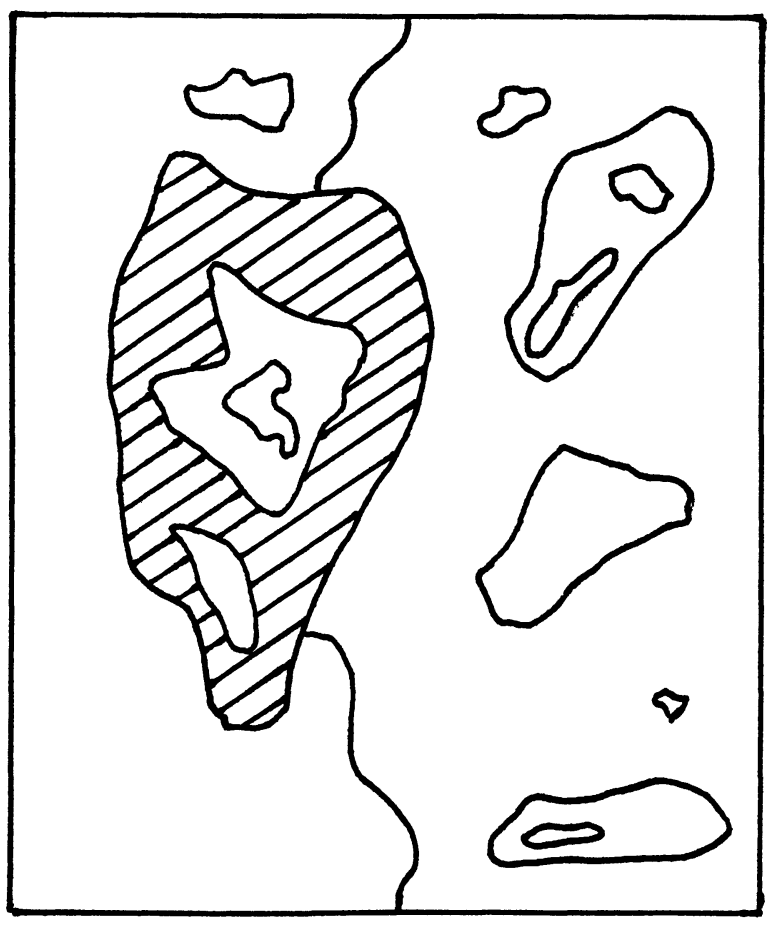

Fig. 1. - A diagram representing $\lambda$-clusters.

ciently low temperature a large region between surfaces, called a $\lambda$-cluster, such as the shaded region with $M$ sites has a superparamagnetic moment of order $M^{1 / 2}$. Imagine for a moment the system is divided into $l \lambda$-clusters each of size $N / l$. Below some freezing temperature each $\lambda$-cluster has a super- paramagnetic moment of order $(N / l)^{1 / 2}$ with combined moment equal to $\mu(N l)^{1 / 2}$ where $\mu=0(1)$ :

$$
N \int_{-b}^{b} \chi_{\mathrm{s}}(B) \mathrm{d} B \leqslant 2 \mu(N l)^{1 / 2} .
$$

If one assumes that $l$ increases less rapidly than $N$, $\int_{-b}^{b} \chi_{\mathrm{s}}(B) \mathrm{d} B$ is less than $0(1)$ so that $\chi_{\mathrm{s}}$ makes no contribution to the susceptibility as measured in a physical experiment :

$$
\chi_{\mathrm{m}}(0)=\chi(0)-\chi_{\mathrm{s}}(0)=(k T)^{-1}\left(1-\mu^{2}\right)
$$

$\chi_{\mathrm{m}}(0)$ is the measured zero field susceptibility and has a singularity at $T_{\mathrm{f}}$ for $\mu$ becomes non zero here. If however $l$ is proportional to $N$ the total superparamagnetic moment is proportional to $N$ so that $\chi_{\mathrm{s}}$ makes its full contribution to the measured susceptibility. No singularity is now expected. It is better to define $\chi_{\mathrm{m}}$ so as to include the entire contribution from $\lambda$-clusters which remain finite as $N$ tends to infinity. In this case $\chi_{\mathrm{s}}$ is zero.

Now make the more general assumption that a certain proportion, $\alpha$ of sites lie in $\lambda$-clusters whoses sizes tend to infinity as $N$ tends to infinity :

$$
\begin{aligned}
\chi_{\mathrm{s}}(0) & =(k T)^{-1} \mu^{2} \alpha, \\
\chi_{\mathrm{m}}(0) & =(k T)^{-1}\left(1-\alpha \mu^{2}\right) .
\end{aligned}
$$

If $\alpha$ is non-zero $\chi_{\mathrm{m}}(0)$ now has a singularity at $T_{\mathrm{f}}$. It is important to notice those clusters whose size tends to infinity as $N$ tends to infinity may tend to infinity less rapidly than $N$. In this case the order parameter as defined by eq. (13) vanishes. In this way it may be possible to reconcile Monte Carlo results on defect surfaces with the sharp peak in the susceptibility which is observed experimentally. In the region containing $N(1-\chi)$ sites covered by finite $\lambda$-clusters some of these $\lambda$-clusters may still be very large. In [19] it is suggested the perimeter of some defect surfaces tends to infinity as $N$ tends to infinity. Let $\Gamma$ be such $\lambda$-cluster, containing $M$ sites with susceptibility $\chi_{\Gamma}(B)$ and superparamagnetic moment $\mu M^{1 / 2}$ at low temperature. By eq. (10) $\frac{\partial^{2 j}}{\partial B^{2 j}} \chi_{\Gamma}(0)$ is $0\left(M^{j}\right)$. This means the higher derivatives of the susceptibility of the whole system with respect to the external field must also be very large. This offers scope to compare theory with experimental evidence. Since the RKKY interaction in a physical system is isotropic there is no reason why all the spins of a large cluster should not swivel round while their orientations remain relatively fixed.

Acknowledgement. - The author would like to thank Dr. N. Rivier for valuable help and advice. 


\section{References}

[1] Aharony, A. and Imry, Y., Solid State Commun. 20 (1976) 899.

[2] Amorphous Magnetism, Vol. II, eds. R. A. Levy and R. Hasegawa (Plenum Press, N.Y.) 1977, article by AharonY, A. and IMRY, Y.

[3] Chalupa, J., Solid State Commun. 22 (1977) 315

[4] Mattis, D. C., Phys. Lett. 56A (1976) 421.

[5] Luttinger, J. M., Phys. Rev. Lett. 37 (1976) 778.

[6] Phase Transitions and Critical Phenomena, Vol. III, eds. C. Domb and M. S. Green (Academic Press, N.Y.) 1974 p. 245 .

[7] Wenger, L. E. and Keesom, P. H., Phys. Rev. B 13 (1976) 4053.

[8] Edwards, S. F. and Anderson, P. W., J. Phys. F 5 (1975) 965.

[9] Stauffer, D. and Binder, K., Z. Phys. B 30 (1978) 313.
[10] Domb, C., J. Phys. A 9 (1976) L-17.

[11] Bray, A. J. and Moore, M. A., J. Phys. C 7 (1977) L-333.

[12] Bray, A. J., Moore, M. A. and Reed, P., J. Phys. C 11 (1978) 1187.

[13] BINDER, K., Festkorperproblem 17 (1977) 55.

[14] Binder, K., Z. Phys. B 26 (1977) 339.

[15] Rapaport, D. C., J. Phys. C 11 (1978) L-111.

[16] Canella, V. and Mydosh, J. A., Phys. Rev. B 6 (1972) 4220

[17] GuY, C. N., J. Phys. F 8 (1977) 1505.

[18] Vannimenus, J. and Toulouse, G., J. Phys. C 10 (1977) L-537.

[19] Reed, P., Moore, M. A. and Bray, A. J., J. Phys. C 11 (1978) L-139. 